> La majorité des protéines sécrétées et membranaires sont synthétisées dans le réticulum endoplasmique où elles sont repliées et assemblées avant d'être transportées. Dans certaines conditions cellulaires, des protéines de conformation anormale s'accumulent dans le réticulum endoplasmique et induisent la réponse UPR (unfolded protein response). Chez les mammifères, la réponse UPR inclut une activation de la transcription de gènes cibles et une profonde inhibition de la traduction, ce qui augmente les capacités de repliement et de dégradation et limite l'arrivée de nouvelles protéines dans le réticulum endoplasmique. Les voies de signalisation intracellulaire déclenchées par ce stress sont très originales : elles activent des protéines transmembranaires du réticulum endoplasmique et mettent en jeu un épissage non conventionnel d'un ARN messager. Lorsque cette réponse adaptative est insuffisante, une réponse apoptotique est déclenchée. Le stress du réticulum endoplasmique et/ou la mort cellulaire parfois induite lors de la réponse UPR semblent intervenir dans la physiopathologie de certaines maladies comme le déficit en $\alpha 1$-antitrypsine et la maladie de Parkinson juvénile à transmission autosomique récessive. <

\section{Le stress \\ du réticulum \\ endoplasmique : \\ adaptation et \\ toxicité}

\section{Michèle Garlatti, Robert Barouki}



les chaperons [1] $(\rightarrow)$. $\varepsilon n$ effet, en interagissant avec les segments hydrophobes exposés des protéines en cours de synthèse ou des protéines mal repliées, les chaperons (BiP/GRP78, calnexine, GRP94...) préviennent les erreurs de repliement. Malgré cet environnement optimisé, des protéines de conformation incorrecte sont produites dans le réticulum endoplasmique. Celles-ci ne sont pas transportées le long de la voie sécrétrice, mais elles sont au contraire retenues dans le réticulum endoplasmique où elles sont soit prises en charge par les chaperons pour acquérir une conformation correcte, soit transportées dans le cytoplasme pour être dégradées par le protéasome ERAD (endoplasmic reticulum-associated degradation). Dans certaines conditions cellulaires, la quantité de protéines de conformation anormale dans le réticulum endoplasmique augmente si fortement que cela sature l'équipement cellulaire en chaperons et en protéines de dégradation ; les protéines incorrectes ont alors tendance à s'accumuler, plaçant les cellules dans une situation de «stress du réticulum endoplasmique ». Les cellules soumises à un tel stress sont capables de 
réagir : c'est la réponse UPR (unfolded protein response) [2]. La réponse UPR a pour but d'augmenter les capacités cellulaires d'élimination des protéines incorrectes, mais elle peut aussi aboutir à la mort de la cellule par apoptose si le stress est trop intense ou de trop longue durée. Chez les mammifères, la réponse UPR possède deux composantes (Figure 2). La première consiste à activer la transcription des gènes codant pour les chaperons résidant dans le réticulum endoplasmique, les protéines de la voie sécrétrice et les protéines du système de dégradation ERAD, afin de rendre la voie sécrétrice plus performante. La seconde composante de la réponse UPR est une répression rapide et intense de la synthèse protéique qui limite l'arrivée de nouvelles protéines dans le réticulum endoplasmique et évite donc de surcharger cet organite. Les voies de signalisation intracellulaire de la réponse UPR sont très originales, elles mettent en jeu, en particulier, l'épissage d'un ARN messager spécifique et plusieurs protéines transmembranaires du réticulum endoplasmique.

\section{Comment provoque-t-on un stress du réticulum?}

La réponse UPR fait généralement suite à l'accumulation de protéines de conformation incorrecte dans le réticulum endoplasmique. L'anomalie conformationelle peut avoir différentes causes. Initialement, les gènes codant pour les chaperons GRP78/BiP, GRP94, protéinedisulfure isomérase et Erp72 ont été caractérisés car leur expression est induite dans une situation de déplétion glucidique, d'où le nom de GRP pour glucose regulated protein [3]. Actuellement, la tunicamycine, un inhibiteur de la $\mathrm{N}$-glycosylation des protéines en cours de synthèse dans le réticulum endoplasmique est largement utilisée pour induire artificiellement un stress du réticulum. La tunicamycine agit en bloquant la synthèse des oligosaccharides et empêche donc l'ajout de ces sucres aux chaînes d'asparagine. D'autres agents sont utilisés expérimentalement : le dithiothréitol qui rompt les ponts disulfures, la bréfeldine A qui bloque le transport des protéines du réticulum vers l'appa$(\rightarrow) \mathrm{m} / \mathbf{s} \quad$ reil de Golgi $(\rightarrow)$, la thapsigargine qui inhibe $2000, n^{\circ} 1$, la pompe à calcium du réticulum, etc. [4, p. 112 5]. Un stress du réticulum endoplasmique peut, toutefois, avoir une origine endogène : toute mutation altérant la conformation protéique ou l'oligomérisation peut bloquer les protéines dans le réticulum endoplasmique et engendrer une situation pathologique qui sera évoquée plus loin dans cet article. Des erreurs de transcription ou de traduction peuvent également causer des problèmes conformationnels. Enfin, une réponse UPR a été observée lorsque la chaîne lourde $\mu$ des immunoglobulines est synthétisée en l'absence de la chaîne légère. En effet, la chaîne $\mu$ a besoin d'interagir avec la chaîne légère pour acquérir sa conformation native [6].

\section{La réponse UPR chez la levure}

La voie de signalisation déclenchée au cours de la réponse UPR a été initialement caractérisée chez la levure Saccharomyces cerevisiae par des approches génétiques. La réponse UPR chez la levure est restreinte à l'activation de la transcription de gènes cibles qui codent pour les chaperons du réticulum endoplasmique, les protéines de transport RE-Golgi, les protéines de glycosylation et les constituants de I'appareil de dégradation $\operatorname{RAD}[7,8]$. Deux mutants déficients dans la réponse UPR ont été à l'origine de la découverte de protéines essentielles dans la transduc-



Figure 1. Schéma de la voie sécrétrice. Les protéines empruntant la voie sécrétrice sont synthétisées par des ribosomes associés à la membrane du réticulum endoplasmique $(R \varepsilon)$. Les protéines en cours de synthèse interagissent avec les chaperons résidant dans le $R \varepsilon$ pour être correctement repliées. Si la conformation est juste, la protéine quitte le RE pour atteindre son site définitif ; si la conformation est incorrecte, la protéine est retenue dans le RE. Ces protéines incorrectes sont prises en charge par les chaperons pour acquérir de nouveau une conformation correcte et suivre la voie sécrétrice, ou sont transloquées dans le cytoplasme pour être dégradées par le protéasome ERAD. 
tion du signal de la réponse UPR : les protéines Irelp et Haclp (Figure 3) $[9,10]$.

\section{Deux acteurs essentiels: Irelp et Haclp}

La protéine Irelp est une protéine transmembranaire du réticulum endoplasmique dont le domaine $\mathrm{N}$-terminal est placé dans la lumière du réticulum endoplasmique et le domaine $\mathrm{C}$-terminal est situé dans le cytoplasme et/ou dans le noyau de la cellule. La protéine Irelp est le chaînon le plus en amont de la voie de signalisation ; son domaine intraluminal semble en effet impliqué dans la détection du stress du réticulum endoplasmique. Le

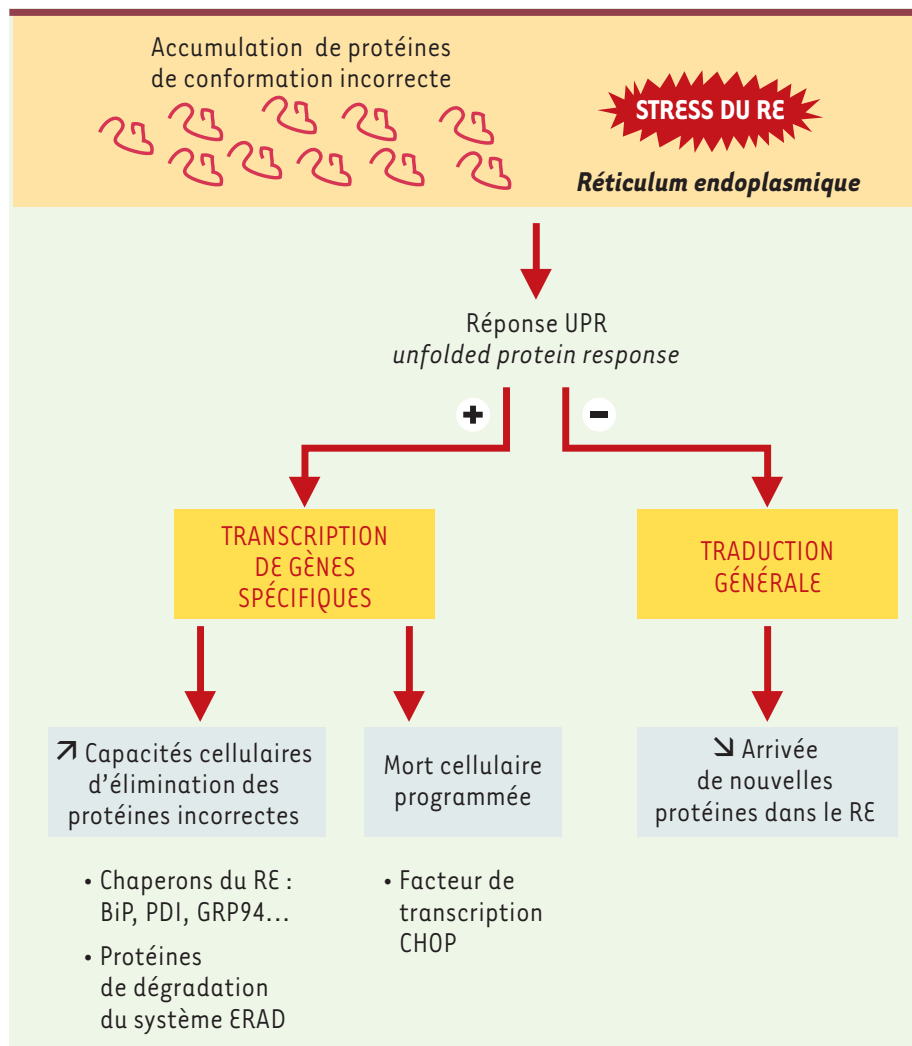

Figure 2. La réponse UPR (unfolded protein response) chez les mammifères. Dans certaines conditions cellulaires, des protéines de conformation incorrecte sont produites en grande quantité dans le réticulum endoplasmique (RE). Lorsque l'équipement cellulaire basal en protéines de repliement et de dégradation est saturé, ces protéines anormales s'accumulent, ce qui est ressenti comme un stress. La cellule de mammifère soumise à ce «stress du RE »a développé la réponse UPR qui agit à deux niveaux. D'une part, elle active la transcription de gènes cibles afin de replier plus efficacement les protéines, d'activer le trafic intracellulaire au sein de la voie sécrétrice et de dégrader plus activement les protéines incorrectes. D'autre part, la traduction est réprimée pour éviter l'afflux de nouvelles protéines dans le RE. Bien que toutes ces réponses tentent de faire survivre la cellule, la réponse UPR peut choisir la mort cellulaire si le stress est trop intense. mécanisme de détection du stress du réticulum endoplasmique a été bien caractérisé chez les mammifères et sera décrit plus loin. Le domaine C-termiendoribonucléase $[9,13]$. Dans des conditions basales, les protéines Irelp sont sous forme de monomères, inactives. En revanche, un niveau anormalement élevé de protéines incorrectes dans le réticulum endoplasmique entraîne l'oligomérisation des protéines Irelp, leur autophosphorylation en trans via le domaine kinase cytoplasmique et la stimulation de l'activité endoribonucléase. La protéine Irelp ainsi activée réalise l'épissage non conventionnel d'un ARNm spécifique, une étape clé de la transduction du signal de l'UPR chez la levure. L'activation de la protéine Irelp par dimérisation et son activité kinase rappellent le mécanisme de réponse de certains récepteurs membranaires sensibles à des ligands externes.

Le second acteur de la voie est la protéine Haclp, un facteur transcriptionnel de type bZIP (basic leucine zipper) appartenant à la famille des facteurs ATF-CREB [14]. Haclp active directement la transcription des gènes cibles de l'UPR et notamment celle du gène Kar2 (qui code pour un chaperon majoritaire du réticulum endoplasmique et dont l'homologue chez les mammifères est BiP/GRP78) en se liant à une séquence promotrice spécifique, UPRE (unfolded protein response element).

\section{Un épissage non conventionnel}

L'activation du facteur de transcription Haclp au cours de la réponse UPR se fait selon un mécanisme très original. En effet, c'est l'épissage de l'ARN messager $\mathrm{HACl}^{\mathrm{n}}$ ( $\mathrm{n}$ : non traduit) en l'ARNm HACl ${ }^{\mathrm{t}}$ ( $\mathrm{t}$ : traduit) qui permet la synthèse du facteur de transcription actif Haclp [14, 15]. Par ailleurs, cet épissage n'est pas conventionnel car les sites de clivage sont différents des séquences consensus classiques et il n'est pas effectué par le splicéosome [16, 17].

$L^{\prime} A R N m \mathrm{HACl}^{\mathrm{n}}$ est produit de manière constitutive. II est présent en quantité relativement constante dans des conditions basales. Cependant, la protéine codée par l'ARNm HACl ${ }^{\mathrm{n}}$ n'est pas détectable car l'ARN HACl ${ }^{\mathrm{n}}$ contient un intron dont la séquence bloque la traduction en empêchant les ribosomes de progresser sur I'ARNm [15, 18]. En cas d'activation de l'UPR, l'intron est éliminé par clivage au niveau de deux séquences spécifiques. L'équipe de Peter Walter a suggéré que l'épissage de l'intron est réalisé par l'activité endoribonucléasique de la protéine Irelp activée. Des expériences réalisées in vitro ont confirmé qu'lrelp est capable de cliver spécifiquement l'ARNm $\mathrm{HACl}^{\mathrm{n}}$ au niveau des séquences décrites [11]. Les fragments 5' et 3' de l'ARNm sont ensuite soudés par la protéine Rgllp 
qui possède une activité ARNt ligase [17]. La nouvelle protéine Haclp codée par l'ARNm $\mathrm{HACl}^{\mathrm{t}}$ a un domaine C-terminal constituant un domaine de transactivation actif différent de celui codé par l'ARNm $\mathrm{HACl}^{n}$. Le facteur de transcription ainsi formé, Haclp, est transloqué dans le noyau où il active la transcription des gènes cibles en se liant aux motifs UPRE. L'épissage de l'ARNm HACl ${ }^{\mathrm{n}}$ est donc l'étape cruciale de régulation de la voie UPR chez la levure.

\section{La dégradation des protéines très endommagées}

Outre l'augmentation des performances de la voie sécrétrice, la levure renforce aussi ses capacités de dégradation des protéines anormales. Un ensemble d'activités enzymatiques est nécessaire à la dégradation des protéines du réticulum endoplasmique ( $(R A D)$. En effet, les protéines défectueuses du réticulum endoplasmique sont tout d'abord transportées dans le cytoplasme par le «translocon »; elles y sont déglycosylées et ubiquitinylées puis dégradées par le protéasome 26S. Le processus ERAD est activé car la transcription des gènes codant pour les différentes protéines impliquées dans ce processus est stimulée [7, 8, 19]. Ce mécanisme permet d'éviter l'accumulation de protéines fortement dénaturées dans le réticulum endoplasmique et leur éventuelle agrégation.

\section{La réponse UPR chez les mammifères}

La réponse d'adaptation au stress du réticulum endoplasmique est plus complexe et variée dans les cellules de mammifères. D'une part, la réponse UPR autorise la survie des cellules dont les protéines sont « réparables » et elle induit l'apoptose des autres cellules. D'autre part, elle contrôle deux fonctions cellulaires : la transcription et la traduction. Enfin, alors qu'une seule protéine transmembranaire du réticulum endoplasmique est mise en jeu dans la réponse traductionnelle, plusieurs protéines transmembranaires permettent la régulation de la transcription.

\section{Activation de la transcription chez les mammifères}

Chez les mammifères, la réponse transcriptionnelle à un stress du réticulum endoplasmique met en jeu deux voies de signalisation impliquant deux familles de protéines transmembranaires : les protéines Irel et ATF-6 (Figure 4). Les gènes cibles de la réponse UPR sont aussi ceux codant pour les chaperons et la voie sécrétrice. La liste des gènes cibles s'est élargie récemment grâce à l'utilisation des puces ADN [20].

\section{Une voie de signalisation partiellement homologue à celle de la levure}

Des homologues des protéines Irelp et Haclp ont été recherchés chez les mammifères. Les résultats indi- quent une conservation partielle du mécanisme de la réponse UPR entre les mammifères et la levure. Deux protéines homologues d'Irelp ont été identifiées chez I'homme et chez la souris : Irel $\alpha$, exprimée de manière ubiquiste, et Irel $\beta$ spécifique de l'épithélium digestif $[21,23]$. Ces protéines transmembranaires du réticulum endoplasmique sont douées d'activités kinase et

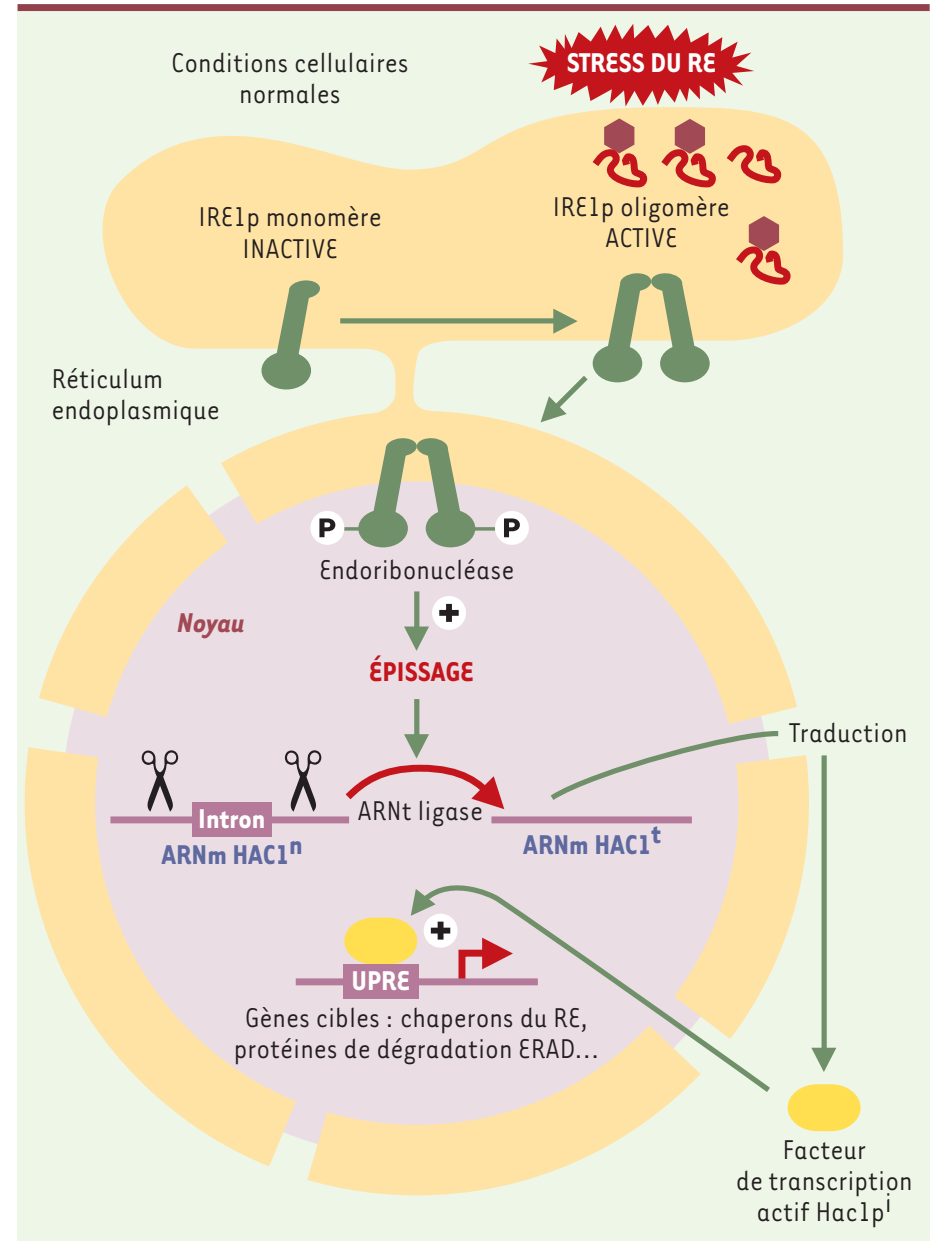

Figure 3. La voie de transduction du signal de la réponse UPR chez la levure met en jeu un épissage non conventionnel. Chez la levure, la réponse UPR ne contrôle que la transcription de gènes. La voie de transduction du signal est bien caractérisée. Dans des conditions cellulaires normales, la protéine transmembranaire Irelp est sous forme de monomère, inactive. Lorsque des protéines dénaturées sont formées dans le réticulum endoplasmique $(R \varepsilon)$, les protéines Irelp s'oligomérisent, ce qui stimule leur activité kinase et engendre leur autophosphorylation en trans. Le domaine C-terminal de Irelp possède une activité endoribonucléase qui est activée par phosphorylation. Elle clive un intron de l'ARN messager $\mathrm{HACl}^{\text {n }}$ ( $\mathrm{n}$ : non traduit) puis l'ARNt ligase Rgllp soude les extrémités 5 ' et $3^{\prime}$ formées. Le nouvel ARNm produit, $\mathrm{HACl}^{\mathrm{t}}$ ( $\mathrm{t}$ : traduit) est efficacement traduit en un facteur de transcription actif, Haclpi. Haclp $p^{i}$ se lie aux séquences promotrices UPRE et active la transcription des gènes codant pour les chaperons du $R \varepsilon$, les protéines de la voie sécrétrice et les éléments du système de dégradation des protéines endommagées (ERAD). 
endoribonucléase dans leur partie cytoplasmique. Le domaine $\mathrm{N}$-terminal de ces protéines, en collaboration avec le chaperon GRP78/BiP, est impliqué dans le déclenchement de la réponse UPR [24]. Ainsi, en l'absence de protéines dénaturées, le chaperon GRP78/BiP est présent à l'état basal dans le réticulum endoplasmique; il interagit avec le domaine intraluminal de lrel et maintient cette protéine à l'état de monomère inactif. Lorsque des protéines incorrectes sont formées dans le réticulum endoplasmique, elles sont reconnues par le chaperon GRP78/BiP qui doit pour cela se dissocier des protéines Irel. Les protéines Irel libérées peuvent s'oli-



Figure 4. La réponse UPR transcriptionelle chez les mammifères : rôle de deux familles de protéines transmembranaires du réticulum endoplasmique : Irel et ATF-6. Dans des conditions cellulaires normales, les protéines Irel $\alpha$ et $\beta$ sont maintenues sous forme de monomères inactives par interaction avec le chaperon majoritaire du réticulum endoplasmique (RE) : BiP/GRP78. En situation de stress du RE, les chaperons BiP se dissocient des protéines transmembranaires Irel pour interagir avec les protéines anormales. Les protéines Irel ainsi libérées s'oligomérisent, s'autophosphorylent en trans, ce qui stimule leur activité endoribonucléase qui clive alors l'ARNm $X B P-1(n)$ (n, non épissé). La trancription du gène XBP-l(n) est activée par les facteurs de transcription p50ATF- 6 $\alpha$ et $\beta$, issus du clivage protéolytique des protéines transmembranaires ATF- 6 induit par le stress du RE. Les ARNm XBP- $1(n)$ sont donc produits en grande quantité en situation de stress, et un intron de 26 bases est clivé par les protéines Irel activées. Le nouvel ARNm XBP-1 (é) (é, épissé) est traduit en un facteur de transcription pXBP-1 capable d'activer la transcription des gènes cibles de l'UPR par liaison à des éléments de réponse au stress du RE, ERSE, qui se lient de manière constitutive aux facteurs de trancription NF- $y$. Les facteurs de transcription p50ATF$6 \alpha$ ou p60ATF6 se lient aussi aux motifs ERSE et activent la transcription des mêmes gènes cibles. gomériser, ce qui stimule leur activité kinase et conduit à leur autophosphorylation en trans. Le cycle de signalisation se termine lorsque GRP78/BiP, produit en excès en réponse à l'induction de son gène, se lie à nouveau aux Irel et inhibe leur dimérisation et leur activation. Ce modèle rend donc compte du déclenche-

cytoplasmique des protéines de mammifères présente une effet, les protéines Irel $\alpha$ et Irel $\beta$ recombinantes et purifiées clivent I'ARNm HACl ${ }^{\mathrm{n}}$ de levure in vitro aux mêmes séquences que la protéine de levure [22, 25]. De plus, la mutation du domaine endoribonucléasique rend ces protéines incapables de déclencher la réponse UPR lorsqu'elles sont surexprimées, ce qui suggère fortement que la voie de signalisation Irel-dépendante des mammifères met aussi en jeu un épissage [26]. Pendant longtemps, le(s) ARNm cible(s) des Irel de mammifère est(sont) resté(s) inconnu(s). Ce n'est que très récemment que les équipes de $K$. Mori et $D$. Ron ont découvert, par une stratégie génétique de mutagenèse chez Caenorhabditis elegans, le rôle essentiel du facteur de transcription XBP- 1 ( $X$ box binding protein-1) dans la réponse UPR des eucaryotes supérieurs [27-29]. Ils ont montré que l'expression de la protéine pXBP-l(é) (é : épissé) est fortement induite par le stress du réticulum endoplasmique et que l'ARNm de XBP- 1 est plus court de 26 bases en situation de stress du réticulum endoplasmique. Le rôle des protéines Irel dans la maturation de l'ARNm XBP- 1 au cours d'un stress du réticulum endoplasmique a été confirmé in vitro par incubation de la protéine purifiée Irel $\beta$ et de I'ARNm 
XBP-1 ( $n$ ) ( $n$ : non épissé). Le clivage de l'intron change le cadre de lecture dans la partie $C$ - terminale de la protéine $\mathrm{pXBP}-1($ é$)$; celui-ci constitue un domaine très actif de transactivation de la transcription. La protéine pXBP-1(é) se lie sur les séquences ERSE (élément de réponse au stress du $R \varepsilon$ ) des promoteurs cibles de I'UPR et induit leur expression.

Les voies de transduction du signal Irel-dépendantes de levure et des eucaryotes supérieurs ont donc un point commun. Dans les deux cas, il y a épissage d'un intron (252 nt pour HACl, 26 nt pour XBP-1 de mammifères), ce qui engendre des facteurs de transcription très actifs. Trois différences sont cependant à noter : il n'y pas de séquence inhibitrice de la traduction dans I'ARN XBP$1(n)$, l'excision de l'intron change le cadre de lecture de I'ARN XBP-l et l'expression du gène XBP- 1 est induite par le stress du réticulum endoplasmique contrairement au gène $\mathrm{HACl}$ dont l'expression est constitutive.

Mais le rôle des protéines Irel dans la réponse UPR des mammifères n'est pas encore clair. L'invalidation du gène Irel $\beta$ (spécifique de l'épithélium digestif) donne des souris viables, qui présentent une prédisposition aux maladies inflammatoires du côlon [21]. Un résultat surprenant a été obtenu avec des fibroblastes embryonnaires Irel $\alpha^{-/-}$. L'invalidation du gène Irel $\alpha$ murin (dont l'expression est ubiquiste) est létale au stade embryonnaire, ce qui montre un rôle important de cette protéine dans le développement [30]. Des fibroblastes, préparés à partir de ces embryons ont été mis en culture. Bien que dépourvus de protéine Irel $\alpha$ et Irel $\beta$ (puisque Irel $\beta$ est spécifiquement présente dans l'intestin), ils sont capables de développer une réponse transcriptionnelle UPR lors de l'exposition à des agents inducteurs de stress. Des résultats similaires ont été obtenus avec des fibroblastes Irel $\alpha^{/-}$et Irel $\beta^{-/-}$. Cela suggère que le stress du réticulum endoplasmique peut activer la transcription des gènes indépendamment des protéines Irel $\alpha$ et $\beta$. Une hypothèse avancée jusqu'à présent était qu'une troisième isoforme Irel existerait et qu'elle prendrait le relais fonctionnel dans ces cellules. Mais il a été récemment mis en évidence que la protéine XBP-1(é) n'est pas produite dans ces cellules exposées aux agents inducteurs de stress [27] ; il est donc probable qu'en l'absence d'Irel $\alpha$ et $\beta$, l'induction des gènes cibles de I'UPR se fasse par une autre voie ; peut-être la voie ATF6 décrite ci-contre.

\section{Une voie de signalisation mettant en jeu un clivage protéolytique}

La caractérisation de la voie de signalisation UPR chez les mammifères a été aussi réalisée par l'étude des facteurs de transcription se liant aux promoteurs des gènes cibles.
II a été ainsi montré que les facteurs de transcription ATF6 appartenant à la famille ATF-CREB activent les gènes cibles lors d'un stress du réticulum endoplasmique.

L'élément de réponse au stress du réticulum endoplasmique (ERSE) a pour séquence consensus CCAAT-N9CCACG, il est nécessaire et suffisant pour l'activation d'au moins trois chaperons majoritaires du réticulum endoplasmique : GRP78/BiP, GRP94 et la calréticuline [31]. Cet élément peut fixer trois types de facteurs de transcription : NF-Y/CBF, une forme clivée des facteurs ATF-6 [31, 32] et les protéines pXBP-1 [31]. N-FY/CBF est un facteur de transcription général ; il se lie au motif CCAAT des \&RSE de manière constitutive. En revanche, les formes clivées de ATF-6, p50ATF- $6 \alpha$ et p60ATF- $6 \beta$ n'interagissent avec le motif CCACG des ERSE qu'en condition de stress du réticulum endoplasmique [33] ; c'est aussi le cas de pXBP-1(é). Pendant longtemps, seule la liaison des protéines ATF-6 recombinantes était visible, mais récemment la liaison de ces protéines a été démontrée à partir d'extraits nucléaires de cellules stressées [34].

Le mécanisme d'activation du facteur ATF- 6 est différent de celui du facteur Haclp de la levure. En effet, l'activation se fait au niveau de la protéine et non au niveau de l'ARNm. Ainsi, à l'état basal, les protéines ATF- $6 \alpha$ et ATF- $6 \beta$ sont exprimées de manière constitutive sous forme de précurseurs transmembranaires du réticulum endoplasmique [35]. A la suite d'un stress du réticulum endoplasmique, ces précurseurs inactifs sont clivés par une protéase [33]. Les domaines cytoplasmiques libérés, p50ATF-6 $\alpha$ et p60ATF-6 $\beta$, composés de domaines de liaison à I'ADN et d'activation de la transcription, sont capables de migrer vers le noyau et d'induire les gènes cibles. Ce mécanisme rappelle l'activation des facteurs SREBP, impliqués dans la régulation du métabolisme du cholestérol. D'ailleurs, une étude récente du laboratoire de Brown et Goldstein montre que les protéases de site 1 et de site 2 (S1P et S2P), capables de cliver le facteur SREBP (sterol responsive element binding protein) $(\rightarrow$ ) sont $(\rightarrow) \mathrm{m} / \mathrm{s}$ impliquées dans le clivage des facteurs ATF-6 1999, $n^{\circ} 1$, [36]. Alors que le mécanisme d'activation de p. 56 ces protéases par le cholestérol est à présent établi, le mécanisme de son activation par le stress du réticulum endoplasmique et les bases de la spécificité de la réponse restent à déterminer.

En conclusion, deux voies de signalisation coexistent chez les mammifères pour déclencher une réponse transcriptionnelle à la suite d'un stress du réticulum endoplasmique ; elles activent deux facteurs de transcription : les formes clivées d'ATF-6 et de PXBP-1. Un lien très fort existe entre ces deux voies puisqu'en cas de stress du réticulum endoplasmique, les facteurs ATF- 6 se lient au promoteur du gène XBP-1 pour activer sa transcription [29]. 
Une différence importante existe : les facteurs de transcription ATF-6, résultant d'une protéolyse, sont rapidement produits, tandis que le facteur de transcription actif pXBP-1 nécessite une induction de son gène et un épissage par Irel. En revanche, XBP-1 devrait fonctionner plus longtemps qu'ATF- 6 puisqu'il active son propre promoteur. Ces deux voies assurent certainement une grande fiabilité et peut-être des niveaux de réponses cellulaires variables en fontion du stress. Enfin, cela pourrait permettre à certaines cellules de l’organisme, spécialisées dans la sécrétion, de possèder une voie sécrétrice très performante ou très spécifique.

\section{Inhibition de la traduction}

Contrairement à la levure, espèce chez laquelle aucune régulation de la traduction n'est observée, la réponse UPR chez les mammifères engendre aussi une inhibition rapide et transitoire de la traduction. Ce blocage général de la traduction a pour but de diminuer l'afflux de protéines dans le réticulum endoplasmique afin d'en éviter la surcharge (Figure 5). L'inhibition de la traduction se fait par l'intermédiaire d'une troisième protéine transmembranaire du réticulum endoplasmique, PERK $[37,38](\rightarrow)$. PERK possède $(\rightarrow) \mathrm{m} / \mathrm{s}$ des homologies structurales avec les protéines Irel $\alpha$ et $\beta \quad 1999, n^{\circ} 3$, et le mécanisme d'activation de l'activité kinase de PERK p. 423 est similaire à celui des Irel de mammifères. En effet, les domaines N-terminaux, intraluminaux, de ces deux protéines sont interchangeables et interagissent avec BiP/GRP78 [24, 39]. Ainsi, au cours d'un stress du réticulum endoplasmique, PERK est libérée du chaperon BiP; elle peut alors s'oligomériser et s'autophosphoryler en trans. PERK appartient à la famille des kinases capables de phosphoryler la sous-unité $\alpha$ du facteur général d'initiation de la traduction elF-2 [38]. Ces kinases sont activées en réponse à des stress cellulaires variables, mais, dans tous les cas, la phosphorylation de elF-2 $\alpha$ sur le résidu sérine 51 empêche la formation du complexe de pré-initiation de la traduction et donc l'interaction de I'ARNm avec la sous-unité $40 S$ du ribosome. De par son mécanisme, l'inhibition de la traduction devrait toucher l'ensemble des protéines cellulaires, mais certaines protéines semblent échapper à cette régulation. En effet, un travail récent portant sur le facteur transcriptionnel ATF-4 montre que la traduction de son messager est activée par le stress du réticulum endoplasmique [40]. En ce qui concerne les gènes induits par le stress, on ne sait pas si leur expression résulte de la forte activation de leur transcription et d'une inhibition partielle de la traduction de leurs messagers ou s'ils échappent à cette régulation.

\section{Vie-mort cellulaire}

Il a été montré que les protéines Irel $\alpha$ et $\beta$, activées par des condiqui bloque la formation du complexe de pré-initiation de la traduction et inhibe la synthèse des protéines. 
tions de stress du réticulum endoplasmique, stimulent la voie des JNK (Jun N-terminal protein kinases) [41]. Cette voie est impliquée dans la décision que prend une cellule de survivre ou de succomber à un stress. Les protéines Irel activent la voie des JNK en recrutant une protéine adaptatrice TRAF-2 à la membrane du réticulum endoplasmique. Cette activation requiert l'activité kinase de Irel $\alpha$ mais est indépendante de l'activité endoribonucléasique.

Les stress cellulaires, et notamment le stress du réticulum endoplasmique, induisent l'expression du gène codant pour le facteur de transcription CHOP. L'invalidation du gène CHOP a montré qu'en condition de stress du réticulum endoplasmique, CHOP induit la mort cellulaire programmée [42].

\section{La réponse $\varepsilon 0 R$ chez les mammifères}

Un autre type de stress du réticulum endoplasmique a été décrit par certains auteurs; il est dû à une surcharge du réticulum endoplasmique en protéines de conformation normale et il déclenche la réponse cellulaire EOR ( $E R$ overload response) [4, 5]. Cette réponse consiste en l'activation de l'expression des gènes de l'inflammation (cytokines...) et du système immunitaire. Elle a été visualisée lors d'une infection virale (adénovirus $83 / 19 K$ ) au cours de laquelle une synthèse importante de glycoprotéines virales a lieu dans le réticulum endoplasmique [43]. La surexpression des chaînes lourdes $\mu$ des immunoglobulines peut aussi induire cette réponse ainsi que des agents produisant des protéines incorrectes. La voie de signalisation aboutissant à la réponse $E O R$ implique une élévation du calcium cytosolique, un stress oxydant et l'activation du facteur transcriptionnel NF- $\kappa B$. Les mécanismes moléculaires de la réponse $\varepsilon O R$ demeurent beaucoup moins bien caractérisés et étudiés que ceux de la réponse UPR.

\section{Stress du réticulum et pathologie}

Comme nous l'avons vu, les réponses aux stress du réticulum endoplasmique font partie des phénomènes physiologiques d'adaptation. Cependant, dans certains cas, il semble que la cellule ne puisse gérer ce stress ou que le stress du réticulum endoplasmique engendre la mort cellulaire. Cela a été suggéré pour des maladies génétiques comme certains cas de déficit en $\alpha 1$-antitrypsine, de mucoviscidose [44] et de maladie de Parkinson [45]. Les mécanismes par lesquels un stress du réticulum endoplasmique serait toxique sont en général toujours inconnus. Il est possible que les capacités cellulaires en protéines chaperons soient parfois dépassées, même après une réponse UPR, par exemple lorsque la mutation d'un gène produit une protéine de conformation anor- male. Mais la réponse au stress peut elle-même présenter des inconvénients : une inhibition prolongée de la traduction peut altérer des fonctions cellulaires importantes, l'élévation du calcium cellulaire et le stress oxydant observés lors de la réponse EOR peuvent être par eux-mêmes à l'origine de phénomènes toxiques. L' $\alpha 1$-antitrypsine ( $\alpha 1-A T)$ est une protéine sérique d'origine hépatique qui fait partie de la famille des serpines ou inhibiteurs de protéases. Le déficit en $\alpha 1-A T$ résulte de mutations du gène qui inactivent l'enzyme. Cette maladie s'accompagne de diverses manifestations pathologiques, dont la principale est un emphysème pulmonaire dû au défaut d'antiprotéase. Mais, chez certains malades, une insuffisance hépatique sévère pouvant nécessiter une transplantation hépatique est associée ; elle pourrait être due à l'accumulation de la protéine mutée dans le réticulum endoplasmique. Les manifestations de cette maladie seraient donc liées d'une part au déficit enzymatique dans le poumon et, d'autre part, à un phénomène de surcharge en protéine anormale dans le réticulum endoplasmique du foie. Reste à savoir pourquoi seule une partie des patients présente des manifestations pathologiques au niveau du foie.

La forme de mucoviscidose due au mutant $\Delta \mathrm{F} 508 \mathrm{du}$ transporteur CFTR (cystic fibrosis transmembrane conductance regulator) se caractérise par une accumulation de la protéine mutée dans le réticulum endoplasmique des cellules productrices. On pense que la protéine mutante est incapable de suivre son cheminement vers la membrane plasmique et que le stress du réticulum endoplasmique provoqué est responsable, au moins en partie, de la toxicité liée à cette mutation.

La conformation des protéines peut aussi être altérée chimiquement, par exemple par un stress oxydant. Le stress du réticulum pourrait donc faire partie des mécanismes par lesquels certains composés chimiques sont toxiques.

La maladie de Parkinson juvénile à transmission autosomique récessive est due à des altérations du gène parkine. La fonction de la protéine parkine a été définie récemment $(\rightarrow)$ : elle a un rôle essentiel dans l'ubiquitinylation et donc la dégradation protéique [45]. La protéine parkine semble intervenir dans la réponse adaptative au stress du réticu$(\rightarrow) \mathrm{m} / \mathrm{s}$ $1998, n^{\circ} 12$, p. 1451 et $2000, n^{\circ} 10$, p. 1112 lum endoplasmique car son expression est augmentée lors de la réponse UPR et elle permet la dégradation des protéines incorrectes. De plus, la surexpression de la protéine parkine protège les cellules de la mort cellulaire induite par un stress du réticulum endoplasmique, contrairement à des mutants incapables d'ubiquitinyler des substrats [45]. Très récem- 
ment, un substrat de la parkine appelé Pael R (parkinassociated endothelin receptor-like) a été identifié : c'est une protéine transmembranaire, probablement couplée aux protéines $G$. Lorsque ce récepteur est surexprimé, il a tendance à mal se replier, à devenir insoluble et à induire la mort cellulaire. La protéine parkine est capable de supprimer la mort cellulaire induite par le récepteur Pael-R; une des conséquences de l'inactivation du gène parkine serait donc d'empêcher la cellule de dégrader efficacement certaines protéines anormales du réticulum endoplasmique.

\section{Conclusions}

Le stress du réticulum est une situation cellulaire ignorée il y a encore quelques années. La cellule est certainement régulièrement exposée à ce type de $(\rightarrow) \mathrm{m} / \mathrm{s} \quad$ stress $(\rightarrow)$, mais elle a su développer des $1999, n^{\circ} 12$, réponses, UPR et $\varepsilon O R$, qui lui permettent p. 1359 d'éliminer les protéines anormales ou trop abondantes du réticulum endoplasmique. Les mécanismes moléculaires de ces réponses commencent à être bien caractérisés, et même si des questions persistent, leurs compréhension aidera sans doute à élucider les causes physiopathologiques de certaines maladies humaines.

Le stress du réticulum endoplasmique entre dans le cadre plus large des «stress conformationnels » qui sont une composante essentielle de pathologies comme les maladies à prion ou certaines maladies neurodégénératives. En effet, ces maladies se manifestent par l'accumulation ou l'agrégation de protéines anormales dans divers compartiments cellulaires ou extracellulaires et les conséquences toxiques de ces «stress conformationnels » dépendent de la localisation du dépôt protéique et de son intensité. Des approches thérapeutiques visant à modifier la conformation des protéines anormales sont actuellement testées pour éviter l'agrégation et la perte d'activité. Ces approches pourraient donc être utiles dans des maladies associées au stress du réticulum. $\diamond$

\section{SUMMARY}

The stress of the endoplasmic reticulum:

\section{adaptation and toxicity}

The majority of surface and secreted proteins are synthesized in the endoplasmic reticulum where they must acquire the right conformation and assemble before being transported. Under specific cellular conditions, unfolded protein can accumulate and initiate the unfolded protein response (UPR). In mammals, this adaptative response includes transcriptional activation of genes

which enhances endoplasmic reticulum folding and endoplasmic reticulum-associated degradation capacities, and translation attenuation to limit further entry of proteins in the endoplasmic reticulum. The intracellular signal transduction cascades are mediated by endoplasmic reticulum transmembrane proteins and involve unconventional mRNA splicing. If this adaptative response is not sufficient, apoptotic death is triggered. The endoplasmic reticulum stress and/or cellular death, sometimes induced during UPR, seem involved in the physiopathology of some disease such as $\alpha 1$-antitrypsine deficiency and AR-JP, a specific form of Parkinson's disease. $\diamond$

\section{RÉFÉRENCES}

1. Wickner S, Maurizi MR, Gottesman S. Posttranslational quality control: folding, refolding, and degrading proteins. Science 1999 ; 286 : 1888-93.

2. Mori K. Tripartite management of unfolded proteins in the endoplasmic reticulum. Cell $2000 ; 101$ : 451-4.

3. Lee AS. Mammalian stress response: induction of the glucose-regulated protein family. Curr Opin Cell Biol $1992 ; 4$ : 267-73.

4. Kaufman RJ. Stress signaling from the lumen of the endoplasmic reticulum: coordination of gene transcriptional and translational controls. Genes Dev 1999 ; 13 : 1211-33.

5. Pahl HL. Signal transduction from the endoplasmic reticulum to the cell nucleus. Physiol Rev 1999 ; $79: 683-701$.

6. Lee YK, Brewer JW, Hellman $R$, Hendershot LM. BiP and immunoglobulin light chain cooperate to control the folding of heavy chain and ensure the fidelity of immunoglobulin assembly. Mol Biol Cell 1999 ; 10 : 2209-19.

7. Friedlander $R$, Jarosch $\varepsilon$, Urban J, Volkwein C, Sommer T. A regulatory link between $\varepsilon R$-associated protein degradation and the unfolded-protein response. Nat Cell Biol 2000 ; 2 : 379-84.

8. Travers KJ, Patil CK, Wodicka L, Lockhart DJ, Weissman JS, Walter P. Functional and genomic analyses reveal an essential coordination between the unfolded protein response and $\varepsilon R$ associated degradation. Cell $2000 ; 101: 249-58$.

9. Cox JS, Shamu CE, Walter P. Transcriptional induction of genes encoding endoplasmic reticulum resident proteins requires a transmembrane protein kinase. Cell 1993; 73 : 1197-206.

10. Mori K, Ma W, Gething MJ, Sambrook J. A transmembrane protein with a cdc2+/CDC28-related kinase activity is required for signaling from the $E R$ to the nucleus. Cell $1993 ; 74$ : 743-56.

11. Sidrauski C, Walter P. The transmembrane kinase Irelp is a site-specific endonuclease that initiates mRNA splicing in the unfolded protein response. Cell 1997 ; 90 : 1031-9.

12. Shamu $C E$, Walter $P$. Oligomerization and phosphorylation of the Irelp kinase during intracellular signaling from the endoplasmic reticulum to the nucleus. EMBO J 1996 ; 15 : 3028-39. 
13. Welihinda AA, Kaufman RJ. The unfolded protein response pathway in Saccharomyces cerevisiae. Oligomerization and transphosphorylation of Irelp (Ernlp) are required for kinase activation. J Biol Chem 1996 ; 271 : 18181-7.

14. Cox JS, Walter P. A novel mechanism for regulating activity of a transcription factor that controls the unfolded protein response. Cell 1996; 87 : 391-404.

15. Kawahara T, Yanagi H, Yura T, Mori K. Endoplasmic reticulum stress-induced mRNA splicing permits synthesis of transcription factor Haclp/Ern4p that activates the unfolded protein response. Mol Biol Cell $1997 ; 8:$ 1845-62.

16. Kawahara T, Yanagi $\mathrm{H}$, Yura T, Mori K. Unconventional splicing of $\mathrm{HACl} /$ ERN4 mRNA required for the unfolded protein response. Sequence-specific and nonsequential cleavage of the splice sites. J Biol Chem 1998 ; 273 : 1802-7.

17. Sidrauski C, Cox JS, Walter P. tRNA ligase is required for regulated mRNA splicing in the unfolded protein response. Cell 1996 ; 87 : 405-13.

18. Chapman RE, Walter P. Translational attenuation mediated by an mRNA intron. Curr Biol 1997 ; 7 : 850-9.

19. Casagrande R, Stern $P$, Diehn M, et al. Degradation of proteins from the $\varepsilon R$ of $S$. cerevisiae requires an intact unfolded protein response pathway. Mol Cell 2000 ; 5 : 729-35.

20. McCracken AA, Brodsky JL. A molecular portrait of the response to unfolded proteins. Genome Biol $2000 ; 1: 10-3$.

21. Bertolotti A, Wang $X$, Novoa I, et al. Increased sensitivity to dextran sodium sulfate colitis in IRElbeta-deficient mice. J Clin Invest 2001 ; 107 : 585-93.

22. Tirasophon W, Welihinda AA, Kaufman RJ. A stress response pathway from the endoplasmic reticulum to the nucleus requires a novel bifunctional protein kinase/endoribonuclease (Irelp) in mammalian cells. Genes Dev 1998 ; 12 : 1812-24.

23. Wang $X Z$, Harding HP, Zhang $y$, Jolicoeur EM, Kuroda M, Ron D. Cloning of mammalian Irel reveals diversity in the $\varepsilon R$ stress responses. EMBO J 1998 ; $17:$ 5708-17.

24. Bertolotti $A$, Zhang $Y$, Hendershot LM, Harding HP, Ron D. Dynamic interaction of BiP and $\varepsilon R$ stress transducers in the unfolded-protein response. Nat Cell Biol 2000 ; 2 : 326-32.

25. Niwa M, Sidrauski C, Kaufman RJ, Walter P. A role for presenilin-1 in nuclear accumulation of Irel fragments and induction of the mammalian unfolded protein response. Cell 1999 ; 99: 691-702.

26. Tirasophon $W$, Lee $K$, Callaghan B, Welihinda A, Kaufman RJ. The endoribonuclease activity of mammalian IRE] autoregulates its mRNA and is required for the unfolded protein response. Genes Dev 2000 ; 14 : 2725-36.

27. Calfon M, Zeng H, Urano F, et al. IREl couples endoplasmic reticulum load to secretory capacity by processing the XBP-1 mRNA. Nature 2002 ; 415 : 92-6.

28. Shen $X$, Ellis $R E$, Lee $K$, et al. Complementary signaling pathways regulate the unfolded protein response and are required for $C$. elegans development. Cell 2001 ; 107 : 893-903.

29. Yoshida H, Matsui T, Yamamoto A, Okada T, Mori K. XBPI mRNA is induced by ATF6 and spliced by IREl in response to $\varepsilon R$ stress to produce a highly active transcription factor. Cell 2001 ; 107 : 881-91.

30. Urano F, Bertolotti A, Ron D. IREl and efferent signaling from the endoplasmic reticulum. J Cell Sci 2000 ;
$113: 3697-702$.

31. Yoshida H, Haze K, Yanag H, Yura T, Mori K. Identification of the cisacting endoplasmic reticulum stress response element responsible for transcriptional induction of mammalian glucoseregulated proteins. Involvement of basic leucine zipper transcription factors. J Biol Chem 1998 ; $273: 33741-9$.

32. Yoshida H, Okada T, Haze K, et al. ATF6 activated by proteolysis binds in the presence of NF-Y (CBF) directly to the cis-acting element responsible for the mammalian unfolded protein response. Mol Cell Biol $2000 ; 20$ : 6755-67.

33. Haze $\mathrm{K}$, Yoshida $\mathrm{H}$, Yanagi $\mathrm{H}$, Yura T, Mori K. Mammalian transcription factor ATF6 is synthesized as a transmembrane protein and activated by proteolysis in response to endoplasmic reticulum stress. Mol Biol Cell 1999; 10 : 3787-99.

34. Yoshida H, Okada T, Haze K, et al. Endoplasmic reticulum stress-induced formation of transcription factor complex ERSF including NF-Y (CBF) and activating transcription factors 6alpha and 6beta that activates the mammalian unfolded protein response. Mol Cell Biol 2001 ; 21 : 1239-48.

35. Haze K, Okada T, Yoshida H, et al. Identification of the G13 (cAMP-responseelement-binding proteinrelated protein) gene product related to activating transcription factor 6 as a transcriptional activator of the mammalian unfolded protein response. Biochem J 2001 ; 355 : 19-28.

36. Ye J, Rawson RB, Komuro R, et al. ER stress induces cleavage of membranebound ATF6 by the same proteases that process SREBPs. Mol Cell $2000 ; 6$ : 1355-64.

37. Harding HP, Zhang $Y$, Bertolotti A, Zeng H, Ron D.
Perk is essential for translational regulation and cell survival during the unfolded protein response. Mol Cell 2000 ; 5 : 897-904.

38. Harding HP, Zhang $Y$, Ron D. Protein translation and folding are coupled by an endoplasmic-reticulumresident kinase. Nature 1999 ; 397 : 271-4.

39. Liu Cy, Schroder M, Kaufman RJ. Ligand-independent dimerization activates the stress response kinases IREl and PERK in the lumen of the endoplasmic reticulum. J Biol Chem 2000 ; 275 : 24881-5.

40. Harding HP, Novoa II, Zhang $y$, et al. Regulated translation initiation controls stress-induced gene expression in mammalian cells. Mol Cell 2000 ; 6 : 1099-108.

41. Urano F, Wang X, Bertolotti $A$, et al. Coupling of stress in the $\varepsilon R$ to activation of JNK protein kinases by transmembrane protein kinase IREl. Science 2000 ; 287: 664-6.

42. Zinszner $\mathrm{H}$, Kuroda $M$, Wang $X$, et al. CHOP is implicated in programmed cell death in response to impaired function of the endoplasmic reticulum. Genes Dev 1998 ; 12 : 982-95.

43. Pahl HL, Baeuerle PA. Activation of NF-kappa B by $\varepsilon R$ stress requires both $\mathrm{Ca}^{2+}$ and reactive oxygen intermediates as messengers. FEBS Lett 1996 ; 392 : 129-36.

44. Kopito RR, Ron D. Conformational disease. Nat Cell Biol 2000 ; 2 : ह207-9.

45. Imai Y, Soda M, Takahashi R. Parkin suppresses unfolded protein stressinduced cell death through its $\varepsilon 3$ ubiquitin-protein ligase activity. J Biol Chem 2000 ; 275 : 35661-4. 